\title{
A Comparison of Cooking Recipe Named Entities between Japanese and English
}

\author{
Yoko Yamakata \\ The University of Tokyo/JSPS \\ 7-3-1, Hongo, Bunkyo-ku \\ Tokyo, Japan 113-8656 \\ yamakata@hal.t.u-tokyo.ac.jp
}

\author{
John Carroll \\ University of Sussex \\ Falmer \\ Brighton, UK BN1 9QJ \\ J.A.Carroll@sussex.ac.uk
}

\author{
Shinsuke Mori \\ Kyoto University \\ Yoshida-Honmachi, Sakyo-ku \\ Kyoto, Japan 606-8501 \\ forest@i.kyoto-u.ac.jp
}

\begin{abstract}
In this paper, we analyze the structural differences between the instructional text in Japanese and English cooking recipes. First, we constructed an English recipe corpus of 100 recipes, designed to be comparable to an existing Japanese recipe corpus. We annotated recipe named entities (r-NEs) in the English corpus according to guidelines previously defined for Japanese. We trained a state-ofart NE recognizer, PWNER, on the English r-NEs, and achieved very similar accuracy and coverage to previous results for the Japanese corpus, thus demonstrating the quality and consistency of the annotations. Second, we compared the r-NEs annotated in the Japanese and English corpora, and uncovered lexical, semantic, and underlying structural differences between Japanese and English recipes. We discuss reasons for these differences, which have significant implications for cross-language retrieval and automatic translation of recipes.
\end{abstract}

\section{CCS CONCEPTS}

- Computing methodologies $\rightarrow$ Information extraction; • Applied computing $\rightarrow$ Annotation;

\section{KEYWORDS}

Cooking recipe named entity, NER, Japanese and English Comparison

ACM Reference format:

Yoko Yamakata, John Carroll, and Shinsuke Mori. 2017. A Comparison of Cooking Recipe Named Entities between Japanese and English. In Proceedings of CEA2017, Melbourne, Australia, August 19, 2017, 6 pages. DOI: $10.1145 / 3106668.3106672$

\section{INTRODUCTION}

In this paper, we analyze cooking instructional texts in Japanese and English recipes, and discover significant differences between them.

Permission to make digital or hard copies of all or part of this work for personal or classroom use is granted without fee provided that copies are not made or distributed for profit or commercial advantage and that copies bear this notice and the full citation on the first page. Copyrights for components of this work owned by others than the author(s) must be honored. Abstracting with credit is permitted. To copy otherwise, or republish, to post on servers or to redistribute to lists, requires prior specific permission and/or a fee. Request permissions from permissions@acm.org. CEA2017, Melbourne, Australia

( 2017 Copyright held by the owner/author(s). Publication rights licensed to ACM. 978-1-4503-5267-3/17/08 ..\$15.00

DOI: $10.1145 / 3106668.3106672$
Cooking recipes are one of the most popular kinds of Web content for people who manage a household or cook for their family. Several millions of recipes can be found on the Web, written in many languages, including English and Japanese. In Japan, Cookpad, the biggest recipe portal site, has more than 2.5 million recipes and 64 million monthly users according to Cookpad's Corporate Presentation $2017^{1}$. Another portal, Rakuten-Recipe, has more than 1.3 million recipes. In the United States, Food.com has a half million of recipes, while 30 million users visit each of Allrecipe and FoodNetWork every month. Most major web search engines including Google and Bing offer a service for recipe search.

Despite the large number of recipes available online, the language barrier means that it is often difficult for someone to find a recipe in their native language that meets all their desired conditions such as favourite flavours, appropriate nutritional balance, ingredients present in the fridge or store cupboard, available cooking time, and so on. However, literal sentence-to-sentence translation of recipes is not guaranteed to result in comprehensible instructions since linguistic structures and recipe writing conventions differ between languages. Such differences would be a problem if a user wanted to select from a set of candidate recipes originally written in different languages, since the different presentational styles would make them difficult to compare.

In this paper, we present a study of named entity annotation of English cooking instructional text, based on previously devised named entity guidelines for Japanese recipes[3]. Through the process of making the original Japanese guidelines fit English recipes and comparing the resulting named entity annotations, we discovered several types of lexical, semantic, and underlying structural differences between Japanese and English recipes.

\section{JAPANESE AND ENGLISH COOKING RECIPES}

\subsection{Examples}

Table 1 illustrates some differences between instructional recipe texts in Japanese and English. The table shows two recipes from the Web: (1) a literal, sentence to sentence translation into English from a Japanese recipe, and (2) an original English recipe. Both recipes are for Bolognese sauce and their actual cooking processes are similar.

There are at least three significant structural differences between these two recipes. Firstly, the Japanese recipe describes the

\footnotetext{
${ }^{1}$ Cookpad Inc. Corporate Presentation FY2017, https://cf.cpcdn.com/info/assets/wpcontent/uploads/20170309164556/CP2017en.pdf (last accessed 28 June 2017).
} 


\section{Table 1: Examples of Japanese and English Cooking Recipes}

(1) Literal translation into English of the Japanese recipe

Title: "Easy Meat Sauce" $a b$

Ingredients (4 servings)

- 200 grams Ground meat

- 1 medium Onion

- 1 can Canned whole tomatoes

- $1 / 2$ Carrot

- 2 clove Garlic

- 3 Shitake mushrooms

- 2 heaped tablespoons Ketchup

- 2 tbsp Japanese Worcestershire-style sauce (or tonkatsu sauce)

- $1 / 2$ large cube *Soup stock cube

- 1 tbsp Flour

- 1 Olive oil (or vegetable oil)

- $1 / 2$ tbsp Sugar

Method:

(1) Blend all the vegetables in a food processor. If you don't have a processor, just finely chop them.

(2) Heat some olive oil in a frying pan and fry the meat. Add the vegetables from Step 1 and sauté.

(3) Add the flour and gently mix. Add the whole tomatoes, $200 \mathrm{ml}$ of water, the soup stock cube, ketchup, sauce and sugar, and let it simmer for 5 minutes.

(4) Season with salt, and it's done.

(5) If you'd prefer it a little thicker, add some more flour.

${ }^{a}$ https://cookpad.com/us/recipes/152926-easy-meat-sauce by "Nyamiane" ${ }^{b}$ In Japan, "Bolognese sauce" is called "meat sauce".

(2) English recipe

Title: "Spaghetti Bolognese for all" $a$

Ingredients (6 servings)

- 1 dessertspoon vegetable oil

- 1 garlic clove, crushed

- 1 onion, finely chopped

- $500 \mathrm{~g}$ beef mince

- 2 carrots, finely chopped

- 1 tablespoon tomato puree

- 1 (400g) tin chopped tomatoes

- $250 \mathrm{ml}$ beef stock

- $1 / 2$ tsp salt

- $1 / 4$ tsp pepper

- $1 / 4$ tsp dried herbs

\section{Methods:}

(1) Heat the oil in a large saucepan and brown the garlic, onions and mince.

(2) Add the carrots and celery cook until tender.

(3) Stir in the puree, stock, tomatoes and herbs. Cover and simmer for 40 minutes.

${ }^{a}$ http://allrecipes.co.uk/recipe/351/bolognese-sauce.aspx by "tammie" preparation processes of the vegetables in step 1, whereas the English recipe describes these processes separately for each ingredient, in the ingredients list. Secondly, the prepared vegetables are called the vegetables from Step 1; it is very common for Japanese recipes to refer to an intermediate food by a step number, whereas in English this is generally called by a name that captures its main characteristic, such as sauce, mixture, dough, stock, pastry and so on. Thirdly, as shown in step 4 of the Japanese recipe, it is common for the completion process to be described as it's done, and often there is a subsequent step. English recipes sometimes conclude with Enjoy!, but this is unusual and no another step follows it.

Even in the case that automatic Japanese-to-English translation gives fluent results, differences in writing style might make it difficult for a user to compare actual cooking procedures. To select one recipe from a lot of candidates, a user must understand such features as how many steps the recipe has (to guess total cooking time and complexity), how many processes to do in parallel (parallel processing needs skill), which steps are mandatory and which steps are optional, and so on. For the two recipes in Table 1, although the Japanese recipe has two steps more than the English one, the actual cooking processes are almost identical because preprocessing processes are included in the ingredient list in the English recipe, and the fifth step in the Japanese recipe is optional.

\subsection{Principles for Comparative Analysis of Japanese and English}

In the Japanese writing system, there are three types of character: 'hiragana', 'katakana', and 'kanji'. Hiragana and katakana (whose characters are phonograms) are mainly used for representing syllables, whereas kanji (consisting of ideograms adopted from Chinese) represents morphemes or whole words. A single word can be written using various combinations of these three types of character. For example, 玉ねぎ (onion) can also be written as 玉葱, た まねぎ,タマネギ, 玉ねぎ, or 玉ネギ; for consistency, when performing comparative analyses, we consider these variants to be different word types.

In English, also for consistency, we consider different forms of the same verb (e.g. preheat and preheated) to be different word types. For the same reason, singular and plural forms of foods and cooking tools are considered to be different word types.

\subsection{Recipe Named Entities in Japanese}

In this paper we extend a named entity (NE) definition originally devised for recipes in Japanese[3]. Unlike the set of NE types commonly used for general text[5], which are person name, place name, organization name, etc, this is an example of a domainspecific NE definition. The best-known definition of this kind is probably for bio-informatics [4] [1]. A domain specific NE definition and an automatic NE recognizer allow us to make detailed comparisons of documents in the domain and to develop intelligent information processing systems.

The problem of identifying NEs in texts is called named entity recognition (NER). NER is a sequence labeling problem and many solutions have been proposed $[2,5$, inter alia]. The tool we used, PWNER [7], is based on pointwise prediction of whether each word either Begins or is Inside or Outside an NE (i.e. to have 
Table 2: Recipe Named Entity (r-NE) Tags

\begin{tabular}{l|l|l}
\hline Tag & Meaning & Remarks \\
\hline \hline $\mathrm{F}$ & Food & Eatable, also intermediate products \\
\hline $\mathrm{T}$ & Tool & Knife, container, etc. \\
\hline $\mathrm{D}$ & Duration & Duration of cooking \\
\hline $\mathrm{Q}$ & Quantity & Quantity of food \\
\hline Ac & Action by chef & Verb representing a chef's action \\
\hline Ac2 & $\begin{array}{l}\text { Discontinuous } \\
\text { Ac (Eng. only) }\end{array}$ & $\begin{array}{l}\text { Second, non-contiguous part } \\
\text { of a single action by chef }\end{array}$ \\
\hline Af & Action by food & Verb representing action of a food \\
\hline At & $\begin{array}{l}\text { Action by tool } \\
\text { (Eng. only) }\end{array}$ & Verb representing a tool's action \\
\hline Sf & Food state & Food's initial or intermediate state \\
\hline St & Tool state & Tool's initial or intermediate state \\
\hline
\end{tabular}

one of the tags $\mathrm{BIO}$ ) through a search for the best sequence of tags under the tag sequence constraints. The tool has state of the art accuracy.

Mori et al. constructed a Japanese recipe corpus consisting of 208 recipes randomly sampled from the Cookpad web site (https://cookpad.com/). Mori et al.'s original r-NE classification defines eight $\mathrm{r}-\mathrm{NE}$ tags for Japanese recipes; in this paper we adopt this classification while adding a further two tags, Ac2 and At, in order to account for additional phenomena that occur in English recipes. Table 2 shows the resulting ten r-NE tags.

\subsection{Recipe Named Entity Corpus in English}

In our research programme, we want to analyze the similarities and differences between the ways in which recipes are written in Japanese and English. To do this, we use Mori et al.'s Japanese recipe corpus and construct a comparable English recipe corpus, consisting of 100 recipes. These recipes were sampled from each category of 'dish type' in the Allrecipes UK/Ireland web site (http://allrecipes.co.uk/) as of December 2016. The sample selection criteria are based on the proportions and rank orders in which recipes are listed within each dish type. The number of the recipes of each dish type in the web site and the corpus are shown in Table 3. We excluded the dish type Cake because it overlaps completely with the other categories Dessert and Sweets.

An English native speaker annotated the 100 recipes according to Mori et al.'s guidelines, using the IOB2 chunking format [6]. Average annotation time was 24 minutes per recipe, including initial training. After annotation, the first author verified the annotation to ensure it adhered to the guidelines. The numbers of recipes in the Japanese and English corpora and the mean numbers of words and recipe named entities per recipe are shown in Table 4 . The numbers of words in recipes do not differ significantly between the Japanese and English corpora, although there are more r-NEs per recipe in the English corpus than in the Japanese one.

\subsection{English r-NE Recognition Accuracy}

In order to investigate the quality and comparability of the English r-NE tagging, we adopt PWNER, which as mentioned above has been used for Japanese r-NE recognition. In our evaluation we applied 10-fold cross validation to the 100 annotated English recipes, training on 80 recipes, adjusting hyper-parameters on 10
Table 3: Numbers of Recipes in allrecipes.co.uk and the English Recipe Corpus (as of December 2016)

\begin{tabular}{l|r|r|r}
\hline dish type & \#webpage & proportion & \#corpus \\
\hline \hline Bread & 953 & $3.1 \%$ & 3 \\
\hline Pies and tarts & 1251 & $4.0 \%$ & 4 \\
\hline Soup & 2046 & $6.6 \%$ & 7 \\
\hline Salad & 1755 & $5.7 \%$ & 6 \\
\hline Main course & 11523 & $37.2 \%$ & 37 \\
\hline Dessert & 3366 & $10.9 \%$ & 11 \\
\hline Biscuits and cookies & 1655 & $5.3 \%$ & 5 \\
\hline Pancakes & 364 & $1.2 \%$ & 1 \\
\hline Breakfast & 1078 & $3.5 \%$ & 4 \\
\hline Sandwiches & 377 & $1.2 \%$ & 1 \\
\hline Starters & 2331 & $7.5 \%$ & 8 \\
\hline Side dish & 2166 & $7.0 \%$ & 7 \\
\hline Sweets & 416 & $1.3 \%$ & 1 \\
\hline Preserves & 423 & $1.4 \%$ & 1 \\
\hline Drink & 1231 & $4.0 \%$ & 4 \\
\hline Cake & $(4284)$ & - & - \\
\hline \hline Total & 30935 & $100.0 \%$ & 100 \\
\hline
\end{tabular}

Table 4: Cooking Recipe Corpora for Japanese and English

\begin{tabular}{l|r|r|r|r|l}
\hline & \multicolumn{2}{|c|}{ Japanese } & \multicolumn{2}{c|}{ English } & \multirow{2}{*}{ t-test } \\
\cline { 2 - 5 } & Mean & $\sigma$ & Mean & $\sigma$ & \\
\hline \#word & 127.9 & 69.1 & 137.5 & 72.1 & -1.12 \\
\hline \#NE & 40.1 & 20.0 & 54.1 & 27.7 & $-5.04^{* * *}$ \\
\hline \multicolumn{6}{|c}{$p<0.001$}
\end{tabular}

Table 5: Recipe Named Entity (r-NE) Recognition Accuracies

\begin{tabular}{l|r|r|r|r|r|r|c}
\hline tag & cov. & ans. & res. & true & prec. & recall & $\mathrm{F}_{1}$ \\
\hline \hline Ac & $85.0 \%$ & 1788 & 1842 & 1648 & $89.5 \%$ & $92.2 \%$ & 90.8 \\
\hline Ac2 & $67.2 \%$ & 66 & 37 & 18 & $48.6 \%$ & $27.3 \%$ & 35.0 \\
\hline Af & $49.5 \%$ & 107 & 58 & 34 & $58.6 \%$ & $31.8 \%$ & 41.2 \\
\hline At & $61.5 \%$ & 12 & 7 & 7 & $100.0 \%$ & $58.3 \%$ & 73.7 \\
\hline F & $76.7 \%$ & 1782 & 1852 & 1581 & $85.4 \%$ & $88.7 \%$ & 87.0 \\
\hline T & $83.2 \%$ & 689 & 671 & 572 & $85.2 \%$ & $83.0 \%$ & 84.1 \\
\hline $\mathrm{D}$ & $57.5 \%$ & 229 & 219 & 201 & $91.8 \%$ & $87.8 \%$ & 89.7 \\
\hline $\mathrm{Q}$ & $63.4 \%$ & 192 & 166 & 128 & $77.1 \%$ & $66.7 \%$ & 71.5 \\
\hline St & $75.0 \%$ & 314 & 290 & 250 & $86.2 \%$ & $79.6 \%$ & 82.8 \\
\hline Sf & $62.0 \%$ & 372 & 336 & 230 & $68.5 \%$ & $61.8 \%$ & 65.0 \\
\hline \hline Total & $\mathbf{7 9 . 7 \%}$ & 5551 & 5478 & 4669 & $85.2 \%$ & $84.1 \%$ & $\mathbf{8 4 . 7}$ \\
\hline
\end{tabular}

cov. $=$ coverage, ans. $=$ \#answer, res. $=$ \#result, true $=$ \#true positive, prec.$=$ precision, rec.$=$ recall,$F_{1}=$ F-measure

recipes, and testing on 10 recipes. The results by tag type and overall are shown in Table 5. Overall F-measure was 84.7, with r-NER coverage of $79.7 \%$. In previous research with the Japanese recipe corpus [8], PWNER trained on 193 Japanese recipes achieved 84.4 F-measure with a coverage of $79.4 \%$, which is very similar to what we obtain for English. In the evaluation of the Japanese recipe corpus, F-measure increased to 86.7 when the amount of training data was doubled, so given the current evaluation results for English we expect a similar improvement if we increase the amount of data. 


\section{GUIDELINES FOR R-NE TAGGING}

\subsection{General Principles}

Linguistic differences required the r-NE tagging guidelines originally formulated for Japanese [3] to be augmented with a small set of general principles for English:

P1: Prepositions and conjunctions are tagged $O$ (i.e. outside an r$\mathrm{NE}$ ), except when they are part of a collocation.

P2: Adverbs and adverbial phrases are tagged $O$ except when they are part of a phrasal verb (as in the first example below).

Ex.1) throw/Ac-B away/Ac-I

Ex.2) $m i x / A c-B$ in $/ \mathrm{O}$ the/O bowl/T

P3: A sequence of words denoting a single action/food/tool in the cooking process is annotated as a single $\mathrm{r}-\mathrm{NE}$.

Ex.3) frying/T-B pan/T-I

Ex.4) bring/Ac-B to/Ac-I the/Ac-I boil/Ac-I

P4: Auxiliary and modal verbs are tagged $\mathrm{O}$.

When applying principle P2, the annotator initially found it difficult to annotate combinations of a verb and a following word that could function as a prepositional adverb. In some cases the following word should be part of an Ac (action by chef) r-NE but in other cases not. The principle was clarified with the extra instructions that such combinations form a single $\mathrm{r}-\mathrm{NE}$ if they pass both of the following two tests:

- If there is a noun phrase immediately after the prepositional adverb, can it be moved to immediately follow the verb?

- Do the verb and prepositional adverb together mean something different to when the words are considered separately?

\subsection{New r-NE Tags for English}

3.2.1 Ac2 (Discontinuous Ac). The tagging guidelines specify that each chef action in the cooking process should be tagged as a single r-NE Ac. In Japanese, words corresponding to a single action are always contiguous. However, in English, a single chef action can be expressed as a discontinuous phrase in the following three situations:

Type 1: phrasal verbs

Ex.5) throw (something) away, set (something) aside, heat (something) through

Type 2: verb/purpose combination

Ex.6) toss (something) to coat, use (something) to make, season with salt to taste.

Type 3: collocations

Ex.7) bring (something) to the boil, set (something) aside

To address such situations, we added a new tag Ac2 which is used to annotate the discontinuous second phrase.

3.2.2 At (Action by Tool). The guidelines for Japanese do not define a tag to represent a tool (in contrast to the chef) carrying out an action. However, there are 12 such cases in 100 recipes in the English recipe corpus. Seven of the 11 Cake recipes and one of the six Bread recipes in the corpus contain phrases similar to the following:

Ex.8) until a skewer inserted into the center comes/At-B out/At-I clean

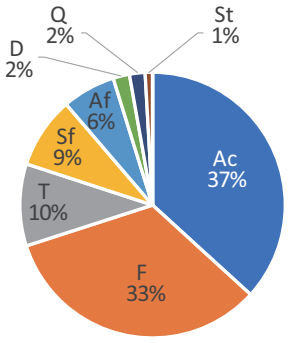

(a) Japanese corpus

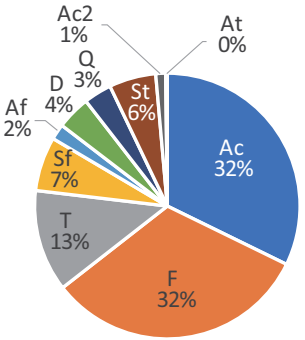

(b) English corpus
Figure 1: Proportions of the $10 \mathrm{r}-\mathrm{NE}$ tag types.

Table 6: The 10 Most Frequent State of Tool (St) Entities..

\begin{tabular}{r|c|l|r|l|r}
\hline \multirow{2}{*}{ rank } & \multicolumn{3}{|c|}{ Japanese (208 recipes) } & \multicolumn{2}{l}{ English (100 recipes) } \\
\cline { 2 - 6 } & word & meaning & freq & word & freq \\
\hline \hline 1 & 180 度 & $180 \mathrm{C}$ & 9 & large & 58 \\
\hline 2 & $170{ }^{\circ} \mathrm{C}$ & $170 \mathrm{C}$ & 6 & medium & 24 \\
\hline 3 & 弱火 & low heat & 6 & $180 \mathrm{c}$ & 18 \\
\hline 4 & 170 度 & $170 \mathrm{C}$ & 5 & small & 16 \\
\hline 5 & $180{ }^{\circ} \mathrm{C}$ & $180 \mathrm{C}$ & 4 & gas mark 4 & 11 \\
\hline 6 & 強火 & high heat & 3 & gas 4 & 10 \\
\hline 7 & 中火 & medium heat & 3 & clean & 10 \\
\hline 8 & $150{ }^{\circ} \mathrm{C}$ & $150 \mathrm{C}$ & 2 & medium-high & 7 \\
\hline 9 & $500 \mathrm{~W}$ & $500 \mathrm{~W}$ & 2 & low & 7 \\
\hline 10 & 200 度 & $200 \mathrm{C}$ & 2 & $190 \mathrm{c}$ & 6 \\
\hline \multicolumn{5}{|l|}{}
\end{tabular}

The remaining four cases of At involve a bread maker, which performs the actions finished, go, heat and signals.

\section{DIFFERENCES IN R-NE DISTRIBUTIONS BETWEEN JAPANESE AND ENGLISH}

Figure 1 shows the proportions of each $r-N E$ tag in the Japanese and English corpora. These proportions are very similar between the two languages, except for St (state of tool) and Af (action by food).

\subsection{Wide difference in St (state of tool)}

The proportion of St r-NEs in the Japanese corpus is smaller than in the English one. The reason is mostly due to differences in word segmentation between the languages. Table 6 lists the 10 most frequently occurring St entities in the two corpora. This shows that in Japanese all of them are related to heating parameters such as temperature, and hob and oven settings.

The third most frequent St entity in Japanese is 弱火, which contains two kanji characters forming a single word, meaning low (弱) heat (火). Depending on context, 弱火 is tagged St or T:

Ex.9) 弱火/St に/O する/Ac (lit. low-heat/St reduce/Ac) = reduce heat to low

Ex.10) 弱火 $/ \mathrm{T}$ で/O 炒める/Ac (lit. low-heat/T fry/Ac) = fry over a low heat

Table 7 shows the numbers and proportions of T and St r-NEs given to the kanji heat words in the Japanese corpus. As shown in the table, these representations are more likely to be tagged T than St; 
Table 7: Tags for kanji heat words

\begin{tabular}{|c|c|c|c|}
\hline \multicolumn{4}{|c|}{ Japanese (208 recipes) } \\
\hline word & meaning & $\mathrm{T}$ & St \\
\hline 弱火 & low heat & 29 & 6 \\
\hline 中火 & medium heat & 13 & 3 \\
\hline 強火 & high heat & 13 & 3 \\
\hline & & $82 \%$ & $18 \%$ \\
\hline
\end{tabular}

this is one of the reasons why the proportion of St is smaller in the Japanese corpus than in the English one.

The first and fourth most frequent words annotated St in the English corpus, large and small, often modify a cooking tool; however, in Japanese a combination of size and name of a cooking tool is generally represented by a single word - for example 小鍋 (small pot) - which would be annotated as T, and there would be no St annotation.

\subsection{Wide difference in Af (action by food)}

The proportion of Af r-NEs in the English corpus is smaller than in the Japanese one. Table 8 lists the 10 most frequent Af entities. The table suggests that one reason for the difference in proportion is how exit/continue/start conditions of Ac (action by chef) are stated. In Japanese, it is very common to express such conditions by a pair of Af and Sf r-NEs as follows:

$$
\begin{aligned}
& \text { Ex.11) やわらか }(=s o f t) / \mathrm{Sf} く / \mathrm{O} \text { な }(=\text { become }) / \mathrm{Af} っ た ら / O \\
& \quad=\text { until soft } \\
& \text { Ex.12) 火 }(=\text { heat }) / \mathrm{Sf} \text { が/O 通 (=through)/Af ったら/O } \\
& \quad=\text { until well cooked }
\end{aligned}
$$

Notably, the word sequence (adjective)/Sf に/O な (=become)/Af る /O appears 89 times in the 208 recipes and accounts for $17 \%$ of all $\mathrm{Sf}$ entities in the Japanese corpus.

In the English recipes, exit/continue/start conditions are expressed using the prepositions until (105), when (6), if (17), once (5), before (23), and after (4) (where (.) indicates frequency). Typical phrases are:

- until Sf

Ex.13) until soft, until combined, until melted, until smooth, until doubled in volume, until sauce thickens

- until $\mathrm{F}$ is/are Sf

Ex.14) until ingredients are well mixed, until the carrots are tender, until the mixture is smooth and creamy

- allow $\mathrm{F}$ to $\mathrm{Af}$

Ex.15) allow to cool, allow steam to escape, allow the potatoes to dry out, allow for it to set

- leave $\mathrm{F}$ to Af

Ex.16) Cover and leave to rise in a warm place for 15 to 20 minutes, leave to absorb, leave to marinate, leave to rest

A further reason for the difference in the proportion of Af is how completion of the cooking procedure is described. 106 of 208 (51\%) recipes in the Japanese corpus refer to completion of the cooking as their final step, but English recipes generally do not do this.

Ex.17) 火/Sf が通っ/Afたら出来上がり/Af

(the food reaches the final state when it is well cooked)
Table 8: The 10 Most Frequent Action by Food (Af) Entities.

\begin{tabular}{r|l|l|r|l|r}
\hline \multirow{2}{*}{ rank } & \multicolumn{3}{|c|}{ Japanese (208 recipes) } & \multicolumn{2}{c}{$\begin{array}{c}\text { English } \\
\text { (100 recipes) }\end{array}$} \\
\cline { 2 - 6 } & word & meaning & freq & word & freq \\
\hline \hline 1 & な & become & 89 & cool & 15 \\
\hline 2 & 出来上がり & Completion & 60 & rest & 4 \\
\hline 3 & 通 & cooked through & 26 & marinate & 4 \\
\hline 4 & できあがり & Completion & 25 & set & 4 \\
\hline 5 & $つ$ & (Many) & 24 & rise & 4 \\
\hline 6 & 出 & (Many) & 21 & thickens & 3 \\
\hline 7 & 完成 & Completion & 14 & run clear & 3 \\
\hline 8 & 混ざ & mixed well & 12 & turned & 3 \\
\hline 9 & なくな & disappeared & 12 & simmer & 2 \\
\hline 10 & O K & OK & 11 & going & 2 \\
\hline
\end{tabular}

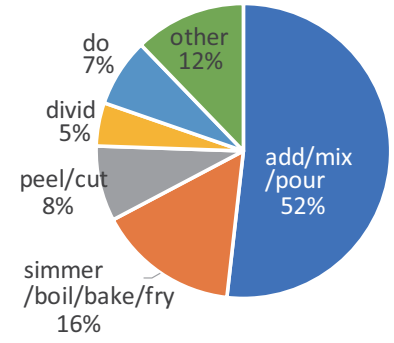

(a) Japanese corpus

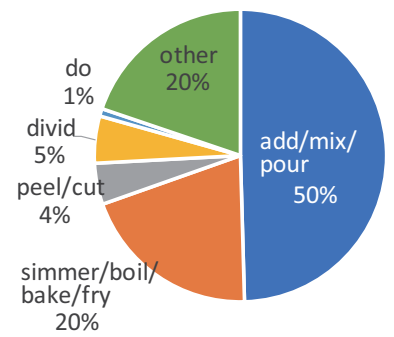

(b) English corpus
Figure 2: Proportions of different types of action by chef (Ac).

A further step can follow the 'completion' step. In the English corpus, $6 \%$ of recipes include the word Enjoy indicating completion; this is always in the final step, except for a single recipe in which advice follows that step.

\subsection{Difference in Ac (action by chef)}

4.3.1 Proportions of Action Types. To find out which types of action are most frequently represented as Ac (action by chef) in each language, we manually classified 185 Ac r-NEs in Japanese and 190 in English, consisting of those Ac r-NEs whose frequency of occurrence accounted for more than $0.1 \%$ of all the Ac entities in each corpus. We classified them into six action types: (1) add/mix/pour, (2) simmer/boil/bake/fry, (3) peel/cut, (4) divide, (5) do, and (6) others. Figure 2 shows the proportions of each action type. As shown in the figure, peel/cut does not appear as frequently in English as in Japanese. The reason is that in English recipes, preparation processes such as chop, dice, mince, grate and melt tend not to be described in the method instructions but are included in the ingredients list - as in the English recipe example in Section 2. Such a representation is also possible in Japanese recipes but is not common.

4.3.2 Ac composed of multiple words. In both the Japanese and English recipes, a single Ac may consist of multiple words.

In Japanese recipes, kanji characters forming a single word can be equivalent to multiple words in English, for example:

Ex.18) 混ぜ込む (mix in), 煮込む (cook well), 流し込む (pour in),

切り分ける (cut into) 
According to the guidelines discussed in Section 3, prepositions and adverbs are annotated as being outside r-NEs except when they form part of a phrasal verb. Therefore, both 混ぜる (mix) and 混ぜ込む (mix in) would be annotated as Ac in the English corpus. Additionally, as mentioned in Section 2.2, kanji always can be replaced by hiragana letters. For example, the kanji word 混ぜ込 む can also be written as 混ぜこむ, まぜ込む and まぜこむ, all of which are pronounced mazekomu.

In English, an Ac r-NE may also be composed of multiple words. One reason is the same as for the Ac2 tag, as discussed in Section 2.2. Additionally, continue (doing) and continue to (do) are typical representations for a single Ac composed of multiple words.

To investigate the diversity of Ac entities we calculated the token-type ratio (TTR). TTR is a widely used measure of lexical diversity and is the number of distinct words (types) divided by the total number of words (tokens). Because TTR is sensitive to document length, we randomly selected 100 recipes from the Japanese recipe corpus in order to match the number of English recipes. In the Japanese recipes, considering Ac r-NEs, there are 351 types and 1472 tokens, giving a TTR of 0.24 . In the English recipes, the corresponding numbers are 372,1842 , and 0.20 ; this indicates a lower diversity in action entities.

\subsection{Difference in F (food)}

Examining the corpora, we devised a classification of foods in recipes which consists of six types:

ingredient: name of an ingredient Ex.19) salt, water, butter, oil, onion, chicken

intermediate: name of an intermediate result Ex.20) dough, mixture, sauce, stock

pronoun:

Ex.21) it, them, all

set: name of a set of foods

Ex.22) ingredients, vegetables, salad

part of: part of a food

Ex.23) liquid, juice, top, centre, base

step number: step number referring to the food resulting from another step

Ex.24) 1, 2

Figure 3 shows the ratios of these six types of $F$ entities in Japanese and English recipes for the top 70\% types (the most frequent 188 food types in Japanese and 124 in English). The figure shows that 'step number' is used more in Japanese, while 'intermediate' is used more in English; these are alternative ways of referring to the food resulting from a previous step. English recipes typically explain how to cook each constituent part separately (such as stock, sauce, and pasta) and then put them together at the end. In this style of instruction, the target of each Ac is generally the result food of the step just before it, and in such cases the target need not be explicitly mentioned in the text. On the other hand, Japanese recipes typically explain how to cook according to the actual cooking order, and then the target food of each Ac is not always the result food of the step just before it. In this case, a result food might come from any previous step, and so it would be referred to by step number.

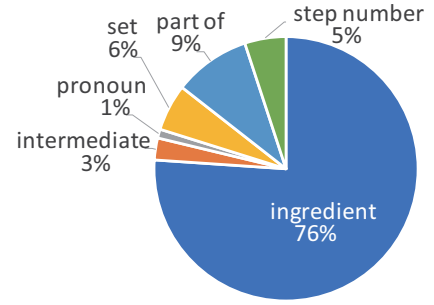

(a) Japanese corpus

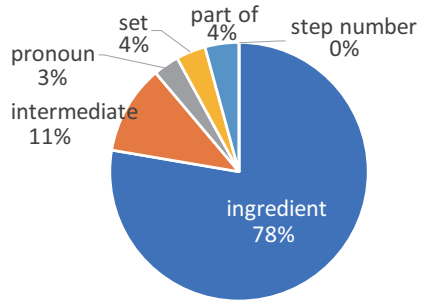

(b) English corpus
Figure 3: Proportions of six types of food representation.

\section{CONCLUSION}

In this paper, we described a new English recipe corpus composed of 100 recipes excerpted from Allrecipes.co.uk, and how we annotated them based on Mori et al.'s r-NE (recipe named entity) guidelines [3]. Then we compared the corpus with an existing Japanese recipe corpus, and discovered several differences in $\mathrm{r}-\mathrm{NE}$ behavior between the two languages. As the results, we discovered several differences including: (1) more than half of the Japanese recipes refer to completion of cooking as their final step, whereas English recipes generally do not do this; (2) there are twice as many peel/cut actions in the Japanese recipes because in English, preparation processes are generally included in the ingredients list; and (3) English recipes refer to the result of a previous step by an intermediate name such as dough or mixture, whereas Japanese uses the step number.

In future work we will build on this initial study to automatically annotate with r-NEs large numbers of Japanese and English recipes, and use these to generate a Japanese-English dictionary specialized for cooking recipes.

\section{ACKNOWLEDGMENTS}

The authors would like to thank Daniel Rolf for his diligent assistance with English recipe named entity annotation. The work is supported by JSPS Grants-in-Aid for Scientific Research Grant Numbers 26280039 and 26280084.

\section{REFERENCES}

[1] Asma Ben Abacha and Pierre Zweigenbaum. 2011. Medical Entity Recognition: A Comparaison of Semantic and Statistical Methods. In BioNLP 2011 Workshop. $56-64$.

[2] Andrew Borthwick. 1999. A Maximum Entropy Approach to Named Entity Recognition. Ph.D. Dissertation. New York University.

[3] Shinsuke Mori, Hirokuni Maeta, Yoko Yamakata, and Tetsuro Sasada. 2014. Flow Graph Corpus from Recipe Texts. In LREC'14.

[4] Lev Ratinov and Dan Roth. 2009. Design Challenges and Misconceptions in Named Entity Recognition. In CoNLL09. 147-155.

[5] Erik F. Tjong Kim Sang and Fien De Meulder. 2003. Introduction to the CoNLL-2003 Shared Task: Language-Independent Named Entity Recognition. In CoNLL03. 142-147.

[6] Erik F. Tjong Kim Sang and Jorn Veenstra. 1999. Representing Text Chunks. In EACL '99. 173-179.

[7] Tetsuro Sasada, Shinsuke Mori, Tatsuya Kawahara, and Yoko Yamakata. 2015. Named Entity Recognizer Trainable from Partially Annotated Data. In PACLING15.

[8] Tetsuro Sasada, Shinsuke Mori, Yoko Yamakata, Hirokuni Maeta, and Tatsuya Kawahara. 2015. Definition of Recipe Terms and Corpus Annotation for their Automatic Recognition (in Japanese). Fournal of Natural Language Processing 22, 2 (2015), 107-131 